\title{
New spectral imaging techniques for blood oximetry in the retina
}

\author{
Ied Alabboud ${ }^{\mathrm{a}}$, Gonzalo Muyo ${ }^{\mathrm{a}}$, Alistair Gorman ${ }^{\mathrm{a}}$, David Mordant ${ }^{\mathrm{b}}$, Andrew McNaught ${ }^{\mathrm{b}}$, Clement \\ Petres $^{\mathrm{a}}$, Yvan R. Petillot ${ }^{\mathrm{a}}$, Andrew R. Harvey*a \\ ${ }^{a}$ School of Engineering and Physical Sciences, Heriot Watt University, Edinburgh, EH14 4AS, UK. \\ ${ }^{\mathrm{b}}$ Cheltenham General Hospital, Department of Ophthalmology, Cheltenham, GL53 7AN, UK.
}

\begin{abstract}
Hyperspectral imaging of the retina presents a unique opportunity for direct and quantitative mapping of retinal biochemistry - particularly of the vasculature where blood oximetry is enabled by the strong variation of absorption spectra with oxygenation. This is particularly pertinent both to research and to clinical investigation and diagnosis of retinal diseases such as diabetes, glaucoma and age-related macular degeneration. The optimal exploitation of hyperspectral imaging however, presents a set of challenging problems, including; the poorly characterised and controlled optical environment of structures within the retina to be imaged; the erratic motion of the eye ball; and the compounding effects of the optical sensitivity of the retina and the low numerical aperture of the eye. We have developed two spectral imaging techniques to address these issues. We describe first a system in which a liquid crystal tuneable filter is integrated into the illumination system of a conventional fundus camera to enable time-sequential, random access recording of narrow-band spectral images. Image processing techniques are described to eradicate the artefacts that may be introduced by time-sequential imaging. In addition we describe a unique snapshot spectral imaging technique dubbed IRIS that employs polarising interferometry and Wollaston prism beam splitters to simultaneously replicate and spectrally filter images of the retina into multiple spectral bands onto a single detector array. Results of early clinical trials acquired with these two techniques together with a physical model which enables oximetry map are reported.
\end{abstract}

Keywords: Blood oximetry, retinal imaging, spectral imaging.

\section{INTRODUCTION}

We report here two distinct and new approaches for recording high-quality spectral images of the ocular fundus with the overall aim of facilitating research and clinical diagnosis of retinal function. Of particular interest is the inference of blood oxygenation in the retinal vessels from a measurement of its absorption spectra.

Spectral imaging entails the recording of the spectrum for each pixel in the scene to yield a spectral data cube. It offers the potential for non-invasive quantification of retinal biochemicals (such as oxy- and deoxy-heamoglobin) and therefore of retinal function; this is a capability that is not possible by other retinal imaging modalities. Several techniques have been reported for direct spectral imaging in which various approaches are taken to multiplex the three dimensional data cube through a two-dimensional detector ${ }^{1}$ (there are of course indirect Fourier-transform techniques to perform spectral retinal imaging but these offer no significant advantages, whereas the requirement for prolonged illumination of the retina with intense broadband light is a distinct disadvantage). Traditionally this involves snapshot recording in two of the dimensions of the spectral data cube and the third dimension is scanned in time-sequence. Notable exceptions to this are a very small number of snapshot techniques: computer tomographic imaging spectrometry (CTIS) ${ }^{2,3}$ which employs a holographic element to spectrally smear images followed by computer algorithms for reconstruction of a spectral data cube; dichroic filters to spectrally demulitiplex an image into a small number of spectral bands on a single detector array $^{4,5,6}$, and the Image Replicating Imaging Spectrometer (IRIS) ${ }^{7}$ as described in this paper.

*a.r.harvey@hw.ac.uk; phone +44(0) 131451 3356; fax +44(0) 1314514155.

6631-22 V. 3 (p.1 of 10) / Color: No / Format: A4 / Date: 6/7/2007 7:39:04 AM

SPIE USE: DB Check, Prod Check, Notes: 
In selecting a technique for spectral imaging of the retina a snapshot approach is desirable for image pre-processing and calibration and patient friendliness. Besides, it allows for the future possibility of real-time spectral imaging. IRIS capabilities offer key advantages for retinal imaging over current snapshot techniques such as the spectral line scanner ${ }^{8}$ and CTIS. The line scanner employs an imaging dispersive spectrometer to form a one-dimensional spectral image along the spatial dimension. The resultant images may be located so as to intersect points of interest on the retina, such as a blood vessel, thereby enabling blood oximetry. However, the narrow field of view presents a significant disadvantage in clinical detection of retinal disease where an extended two-dimensional field of view is desirable. On the other hand, CTIS enables two-dimensional field of view, but it is limited by inefficient usage of both the detector array and its large number of spectral bands when only a few is required. In contrast, IRIS can record contiguous bands with high efficiency. Spectrally separated bands can be obtained by placing a tiled array of bandpass filters (mosaic) at the detector plane; this does not affect the pixel efficiency.

Although a snapshot approach such as IRIS is desirable for clinical applications as we present here, a time sequential technique can be very useful as a research tool to investigate the spectral signatures of the retina. To this end, we have also chosen to work with a technique where a series of two-dimensional spectral images are recorded with timesequential scanning of the spectrum by switching of a liquid crystal tunable filter (LCTF). This enables random access of the spectral bands and full-frame detector usage. There are, however, additional problems associated with this approach that prevent from obtaining accurate measurements, namely calibration (since no two images have identical illumination) and misregistration (due to random movement of the eyeball). In the first section of this paper, we describe a time-sequential spectral fundus camera together with new algorithms for coregistration and calibration of the data cube that allow us to obtain improved oxygen measurements. Fundamentally, these new algorithms provide enhanced optical density estimations of the whole retinal vessel tree. We show that this technique is an adequate approach to acquiring a wide-field spectral data cube for research and clinical exploration because it offers a highly flexible means of identifying wavebands for which the most useful spectral information exists.

Finally, in the last section, we report on the development of an IRIS retinal camera that records a spectral data cube directly onto a conventional detector array. The snapshot spectral capability removes the fundamental problems associated with time-sequential techniques which affect accurate blood oximetry. In contrast to the technique mentioned above, IRIS has fixed spectral bands which must be selected accordingly for each specific application.

\section{TIME-SEQUENTIAL SPECTRAL IMAGING OF THE RETINA}

\subsection{Experimental set-up}

The spectral data cube was assembled by scanning the retina for each wavelength and recording it onto a twodimensional detector. A commercial fundus camera (Canon, CF-60z) was modified to accommodate a liquid crystal tuneable filter ${ }^{9}$ (LCTF) into the illumination path, as shown in Figure 1. The LCFT was used to enable wavelength selection from $400 \mathrm{~nm}$ to $700 \mathrm{~nm}$ (7-9nm of spectral bandwidth). Due to the LCTF, the intensity of the filtered light was effectively reduced $50 \%$ compared with the unfiltered light; thus, increasing the safety and patient comfort. The output image from the unmodified fundus camera was re-image to enable the use of the full field of view (60 degrees) with magnification factor of four. For data acquisition and instrument control a program running under Labview was developed. Different flash intensities equipped in the original camera system were used to compensate the variation in the spectral transmission caused by the eye and LCTF components.

The original camera included two axial amplitude masks to block the specular reflections from the two surfaces of the objective lens. After inserting the filter into the optical path, the position of the masks had to be changed and that caused a fixed pattern artefact in the recorded images. A linear polarizer was incorporated in front of the CCD to reduce the impact of the artefact on the recorded images. 


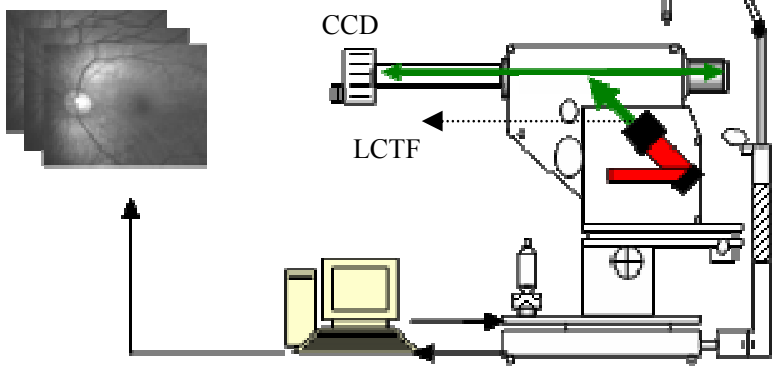

Figure 1.Set-up of time sequential retinal imager, LCTF has been inserted into the optical path of fundus camera.

\subsection{Acquisition, pre-processing and calibration of spectral retinal images}

Spectral retinal images were recorded from 500nm to $700 \mathrm{~nm}$. Patients were advised to fixate on a LED provided with the camera system in order to reduce as much as possible the random movement of the eye. The image of a black target (given by $I_{b}$ ) taken at each wavelength $\lambda$ was subtracted from corresponding the retinal image (given by $I_{R}$ ) to remove the dark current and the artefacts produced by the surface of optical components. An automated algorithm performed this operation, which can be expressed mathematically by

$$
I_{R^{\prime}}(\lambda)=I_{R}(\lambda)-\alpha(\lambda) I_{b}(\lambda)
$$

where $\alpha(\lambda)$ is a wavelength-dependent variable optimised to minimize the artefact.

Due to the nature of the adopted time-sequentional technique, random eye movement and non-uniform illumination affect accurate construction of the retinal spectral cube. Translational and rotational misalignments in the geometry among the recorded spectral images introduced by random eye movement can be corrected by applying an automated coregistration algorithm. As it will be shown, spectral images of the retina vary considerably in appearance from wavelength to wavelength, thus it is important to apply the co-registration algorithm only to those features that remain practically unchanged (like the blood vessels tree) throughout the wavelength range. A relatively simple two dimensional cross-correlation function was found ${ }^{10}$ to enable accurate co-registration between two filtered images, one of them will be used as a reference. Prior to cross-correlation calculation, the recorded images were passed through different types of spatial filters (band-pass filter, matched filter and edge detection with fixed parameters) to facilitate extraction of signatures.

As an example, a typical calibrated and co-registered set of images taken from a spectral data cube is shown in Figure 2 for wavelengths between $580 \mathrm{~nm}$ and $600 \mathrm{~nm}$. In this wavelength range, the optical depth of oxygenated haemoglobin changes from being less than to being greater than the thickness of the larger retinal arteries. The reflectance spectrum of different locations across the retina is dominated by oxygenated and de-oxygenated haemoglobin in both choroidal and retinal vessels, and also by melanin components which vary depending on subjects and ethnicity. As it can be observed from the images in Figure 2, the main components of the retinal images at different wavelength are veins, arteries, the optic disc, the retinal nerve fibre layer and the fovea. Due to the oxygenation state of blood and the depth at which scattering occurs veins and arteries differ in appearance depending on the illumination wavelength. Both veins and arteries appear dark in wavelength range 560-580nm, while in the range 580-600nm arteries start to be translucent but veins still opaque. 


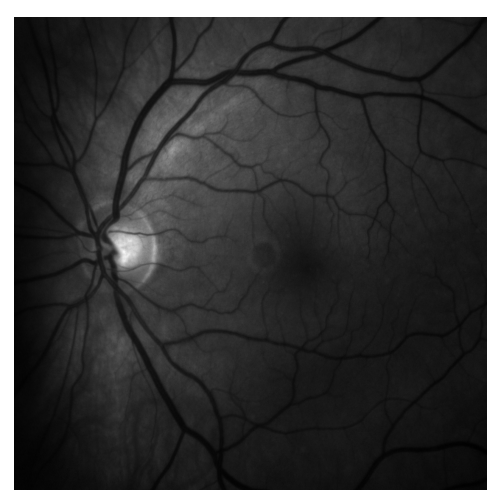

$580 \mathrm{~nm}$

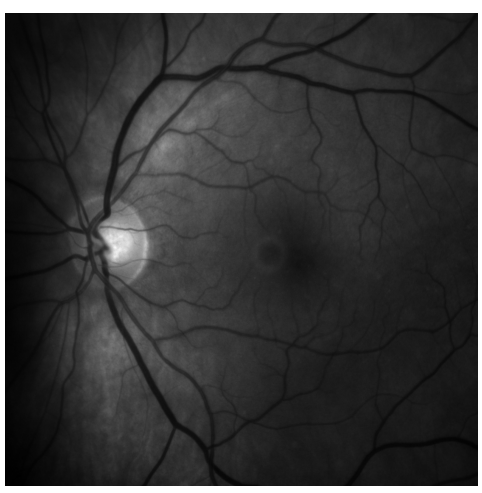

$590 \mathrm{~nm}$

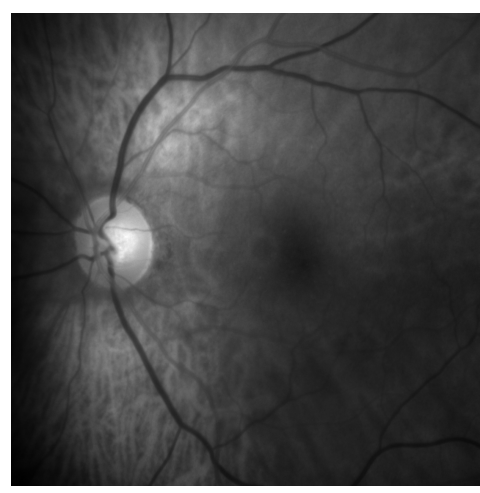

$600 \mathrm{~nm}$

Figure 2.Co-registered and calibrated retinal images $(580-600 \mathrm{~nm})$ from the left eye of healthy subject

\subsection{Retinal oximetry}

After collecting and processing the retinal data cube in the wavelength range from $500 \mathrm{~nm}$ to $700 \mathrm{~nm}$ of healthy subject, an evaluation of the blood oximetry in the retinal vessels was performed.Measuring oxygen saturation in the fundus of the human retina aids the diagnosis and monitoring of disorders for early detection of some diseases. Retinal oximetry was firstly introduced by the work of Hickham \& Frayser ${ }^{11}$ in 1963. Delori ${ }^{12}$ developed the earliest scanning oximeter by using monochromatic beams to scan the vasculature of the retina. A physical model to measure oxygen saturation in retinal vessel was produced by Schweitzer ${ }^{13}$.

In this section, we report qualitative and quantitative methods to measure the oxygen saturation in both retinal veins and arteries.

For qualitative oximetry, retinal images can be mapped depending on the spectra of oxygenated and de-oxygenated blood in the retinal vessels by means of linear spectral unmixing ${ }^{14}$. This processing method employs the spectra of the purest materials or endmembers in the scene (in this case, oxygenated and de-oxygenated blood) to produce a wellclassified image. The model assumes that the spectrum of each pixel in the data cube can be decomposed into a linear combination of the endmembers. Thus, for $m$ endmembers the pixel spectrum $p$ can be expressed mathematically as:

$$
p=\sum_{j=1}^{m} \alpha_{j} E_{j}+N
$$

where $\boldsymbol{E}_{\boldsymbol{j}}$ is the $l$-dimensional endmember vector, $\alpha_{j}$ is the abundance coefficient and $N$ is a noise vector. Two constraints must be placed on $\alpha_{j}$ to obtain realistic physical results:

$$
\sum_{j=1}^{m} \alpha_{j}=1 \text { and } \alpha_{j} \geq 0
$$

Linear unmixing was applied to a set of spectral retinal images from $500 \mathrm{~nm}$ to $650 \mathrm{~nm}$ within the data cube. The final classified images are shown in Figure 3 for the left and right eyes of a healthy subject (images are displayed in colour). It can be observed that the retinal and choroidal vasculature can be mapped as a function of the oxygenation levels when the endmembers corresponding to blood in veins and arteries are selected. Oxygenated (96\% oxygen saturation) and deoxygenated blood (55\% saturation) are represented by red and blue respectively. The intermediate oxygen levels in retinal vessels are given by a linear combination of these two colours. Note that also the choroidal structure has been classified and is clearly apparent in the images. The distinctive areas of blur in the classified images are currently being investigated, although there are indications that the blur may be produced by variations in the absorption of the retinal pigment epithelium. 


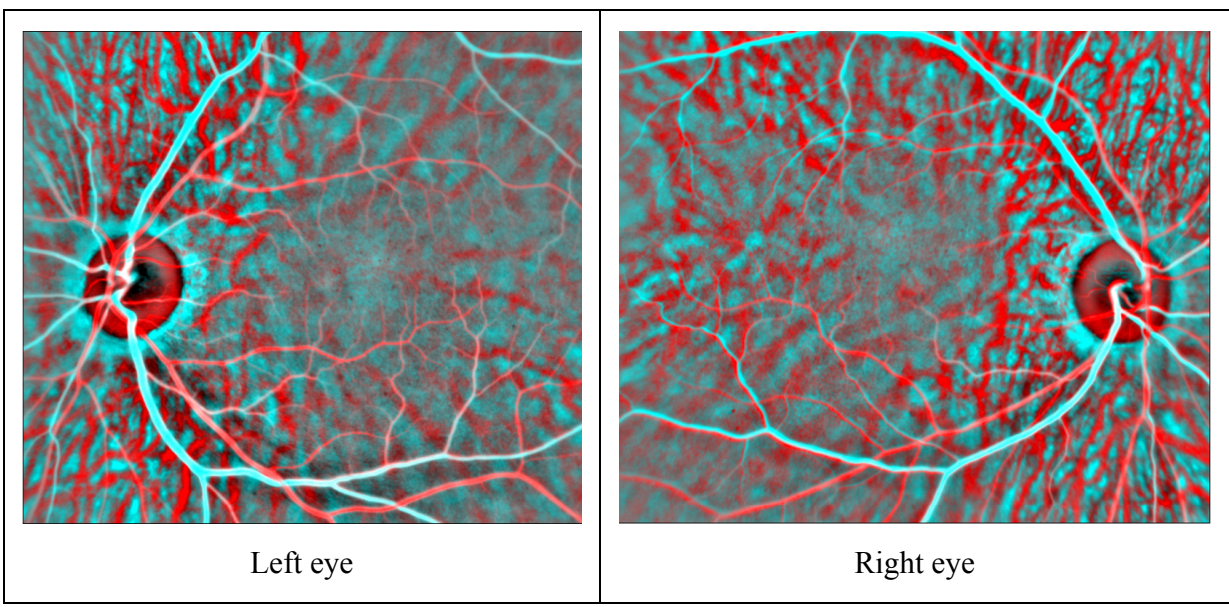

Figure 3. Qualitative oximetric map of the whole retina taken from healthy subject left and right eye, retinal vasculature are mapped according to different oxygenation levels in both veins (blue) and arteries (red). Images displayed in colour.

Although spectral processing methods such as linear unmixing can be used to produce semi-quantitative high-quality classified oximetric maps of the whole retina, more elaborate techniques that take into account physical models of light propagation are required to obtain reliable quantitative oxygen measurement. To this end, we have applied a physical model based on Lambert-Beer's law ${ }^{15}$ to perform quantitative oximetry. This law is only valid for haemolysed blood in a controlled environment; therefore several modifications must be made in order to provide a realistic description of the light propagation in the retina. Initially, for a given illumination wavelength $\lambda$, Lambert-Beer's law can be expressed in terms of the optical density $(O D)$ as

$$
O D(\lambda)=c_{H B \text { total }} d\left[\left(\varepsilon_{\text {oxy }}(\lambda)-\varepsilon_{\text {de-oxy }}(\lambda)\right) O S+\varepsilon_{\text {de-oxy }}(\lambda)\right]
$$

where $c_{\text {HBtotal }}$ is the total concentration of haemoglobin, $\varepsilon_{o x y}$, and $\varepsilon_{d e-o x y}$ are the extinction coefficients ${ }^{15}$ of both oxygenated and deoxygenated haemoglobin, $d$ is the vessel diameter and $O S$ is the oxygen saturation. Clearly, the oxygen saturation can be readily obtained from Eq.(3) by measuring the optical density and vessel diameter in the recorded spectral images. Other parameters in Eq.(3) such as $c_{H B t o t a l}$ and $\varepsilon$ can be easily found in the literature. The variation of the extinction coefficients $\varepsilon$ with wavelength is illustrated in Figure 4

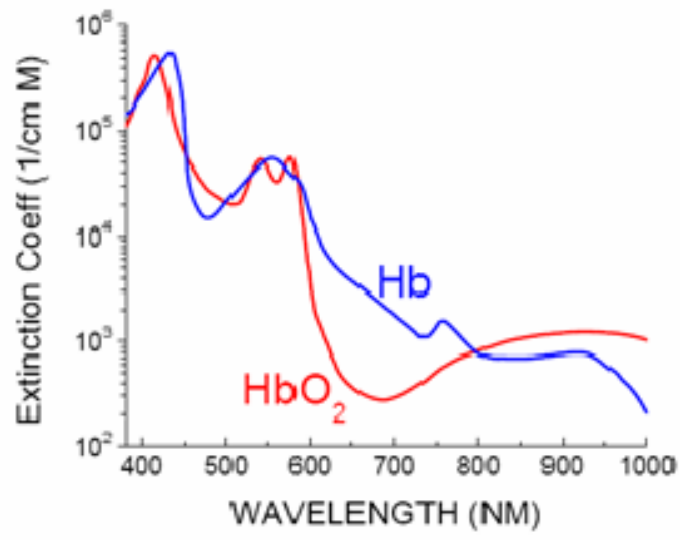

Figure 4. Absorption spectrum of haemoglobin in its oxygenated $\left(\mathrm{HbO}_{2}\right)$ and deoxygenated $(\mathrm{Hb})$ forms.

When considering the whole blood in retinal vessels, other factors like light scattering and multi-optical paths must be taken into account by the physical model. This requires a modified Lambert-Beer's law, thus Eq.(3) now becomes ${ }^{12}$ 


$$
O D(\lambda)=S(\lambda)+\eta c_{H B t o t a l} d\left[\left(\varepsilon_{\text {oxy }}(\lambda)-\varepsilon_{\text {de-oxy }}(\lambda)\right) O S+\varepsilon_{\text {de-oxy }}(\lambda)\right]
$$

where $S$ is the scattering by blood vessels and $\eta$ is the effect of multi optical path ${ }^{16}$ (i.e. $\eta=1$ single path, $\eta=2$ double path). A non-linear fitting of the physical model in Eq.(4) can be applied to the experimental optical densities to obtain quantitative oxygenation measurements. We have developed an algorithm which enables calculating the experimental optical densities along the centre of each retinal vessel in addition to the magnitude of vessel diameter. The algorithm has been applied to each vessel profile and repeated for each wavelength $\lambda$. The various steps of the algorithm can be summarised as follows (a flow chart is shown in Figure 5):

1. Vessel tracking algorithm with a fast-marching based technique ${ }^{17}$ has been used to extract a skeleton of the blood irrigation network of the retina.

2. Extraction of vessels profiles across each tracked vessel.

3. Estimation of the incident light (grey curve) for each vessel profile (black curve) and calculation of the transmission and optical densities profiles.

4. Non-linear fit of the experimental optical densities profiles (grey curve) to produce the estimated optical densities (black curve) in the centre of each vessel profile.

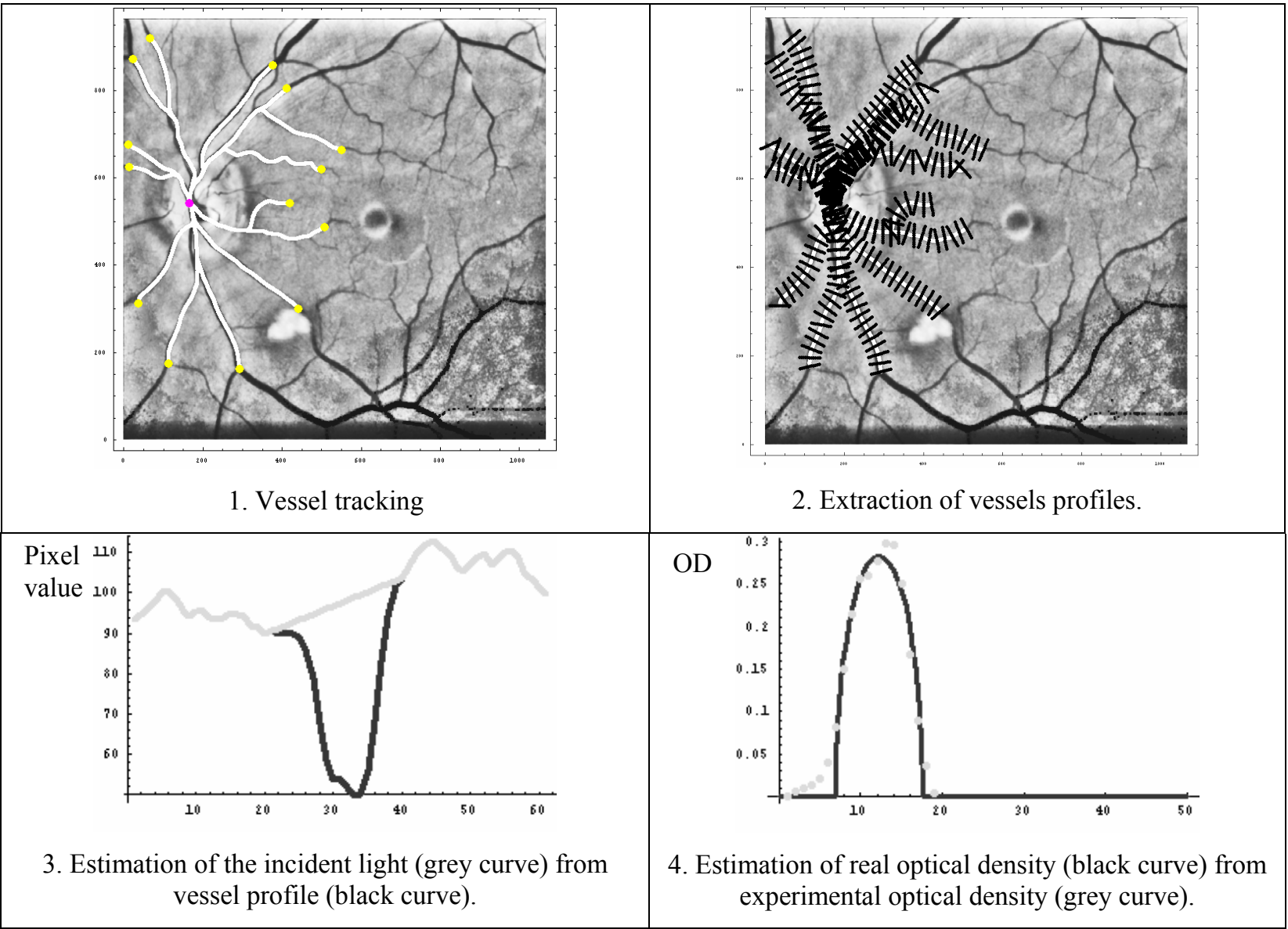

Figure 5. A flow chart of automated algorithm to calculate the optical densities along each tracked vessel.

Non-linear fit of the physical model described in Eq.(4) was applied on the estimated optical densities. The non-linear fit assumes that the scattering $S$ is constant in the selected range whilst the other unknown parameters such as $\eta, c_{H B t o t a l}, d$ and oxygen saturation $O S$ are varied until the modelled optical density values meet the best fit with the estimated ones. This operation was applied to the centre of all the selected retinal vessels. An example of the non-linear fitting applied to 
an artery is shown in Fig.(6) (left). It can be observed that both physical model and experimental data give the best match when the oxygen saturation is $96 \%$, which is in agreement with the expected value. Our oximetry model was also applied on venule blood, as shown in Fig.(6) (right). In this case, the oxygen saturation obtained was 55\%, which can be considered an acceptable value for de-oxygenated blood. Current work is being undertaken to experimentally validate and refine the oximetry model presented here. This involves the construction of an artificial eye that will allow us to extract the spectrum of oxygenated and de-oxygenated of whole blood as opposed to haemolysed blood.

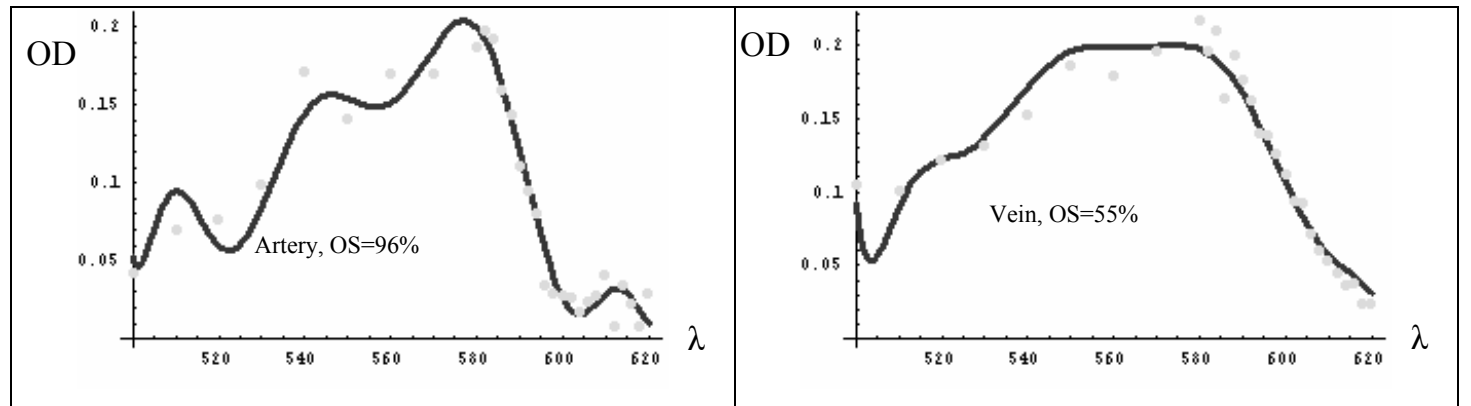

Figure 6.Non-linear fit of the physical model (black curve) to the experimental optical density (grey dots) for blood oximetry on arteries (left) and veins (right).

\section{SNAPSHOT SPECTRAL RETINAL IMAGING}

We have shown that one solution to recording the required three-dimensional data cube using a two-dimensional detector array is to record images in time sequence in a way that scans one of the cube dimensions: a sequence of narrow-band images are recorded and subsequently co-registered. The time sequential nature is undesirable: the increased time required to record the data combined with the infirmity that is common of patients with eye disease is problematic -both for the patient and in the impact on image quality; spectral calibration and image co-registration can be highly problematic and it is not possible to record en face time-resolved spectral images. Unfortunately a putative twodimensional spectral camera; the spectral imaging equivalent of conventional RGB colour camera has been notable by its absence. We report here on the development of a novel two-dimensional snapshot retinal camera that records a spectral data cube directly onto a conventional detector array and requires no complicated inversion algorithm to retrieve the spectral information. The snapshot capability removes the fundamental problems associated with time-sequential techniques which affect accurate blood oximetry.

The key component of this unique retinal camera is an image replicating imaging spectrometer (IRIS) that employs polarising interferometry and Wollaston prism polarising beam splitters to simultaneously replicate images of the retina in multiple spectral bands onto a single detector array. In principle the technique is $100 \%$ optically efficient enabling the intensity of light at the retina to be minimised. A complete description of IRIS can be found in Ref.[18] and Ref.[19]. Basically, IRIS consists of $N$ Wollaston prisms and $N$ retarders that produce $2^{N}$ replicated spectral images. The IRIS birefringent elements are assembled into a common block with index matching fluid to reduce stray reflections and to reduce the effect of non-flatness of the birefringent elements.

This snapshot spectral imaging capability can be readily added to a conventional retinal camera. We report here proof-ofconcept experiments in which eight-band IRIS systems have been integrated into a Discam fundus camera ${ }^{20}$, see Figure 7. The integration of IRIS system into any fundus camera is a straightforward process: the input object plane of IRIS is located at the output image plane of the retinal imager; thus the output image is then spectrally demultiplexed into eight narrow-band images recorded on a cooled CCD detector array. 


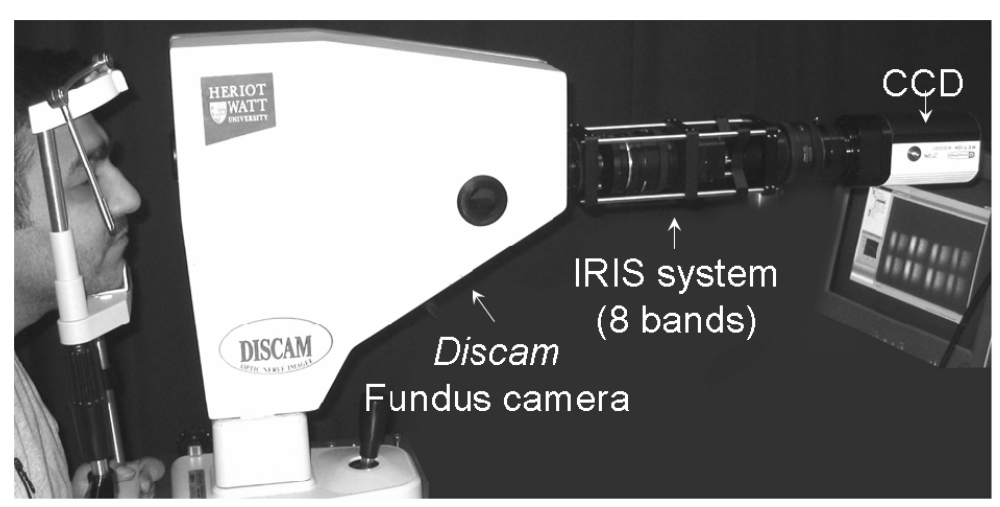

Figure 7. Eight-band IRIS system integrated into Discam retinal camera.

IRIS transmission bands may be optimised for operation in specific spectral regions; in this study, IRIS spectral bands have been appropriately optimised for blood oximetry by means of the absorption spectrum of haemoglobin which is the dominant factor determining the reflectivity of blood vessels. The spectrum of haemoglobin in its oxygenated and deoxygenated forms is shown in Figure 4. Previous research ${ }^{21}$ has shown that an appropriate spectral window for blood oximetry occurs from $560 \mathrm{~nm}$ to $600 \mathrm{~nm}$. Also, the retinal blood's extinction coefficient experiences the maximum variations with oxygenation and two isobestic points (desirable in the physical model that yields a value for oxygen concentration and presented in the previous section). Monitoring the optical density of blood at a range of wavelengths between 560 and $600 \mathrm{~nm}$ enables the effect of blood oxygenation to be separated out from variability introduced by other pigments, such as melanin, to enable blood oxygenation to be calculated.

Two IRIS systems have been designed and assembled with quartz plates with thicknesses optimised to generate eight spectral bands in the ranges $560 \mathrm{~nm}$ to $600 \mathrm{~nm}$ and $577 \mathrm{~nm}$ to $600 \mathrm{~nm}$. The transmission bands modulated by spectral blood-transmission according to Lambert-Beer's law are shown in Figure 8. Note that some bands are optimised to maximise the spectral separation between oxygenated and deoxygenated blood, others produce retinal images practically insensitive to oxygen saturation (isobestic bands). An unusual aspect of IRIS is that the spectral transmission bands are not quite orthogonal in spectral space, but this issue is not significant relative to the high signal-to-noise ratio and snapshot characteristic.

A proof-of-concept demonstration using a pre-existing IRIS system is shown in Figure 9, in which eight replicated narrow-band images were recorded in a single snapshot. The variations of the grey levels are due to the spectral filtering functions of the IRIS system. Notice the clear discrimination between veins and arteries due to the systematic variation of optical density as a function of wavelength. Clearly, the snapshot spectral capability enables improved oxygen measurements in the retina by eradicating calibration and misregistration problems as reported in the time-sequential technique. An exhaustive study together with oximetric results of the retinal vasculature acquired with this spectral snapshot technique will appear in a future paper 


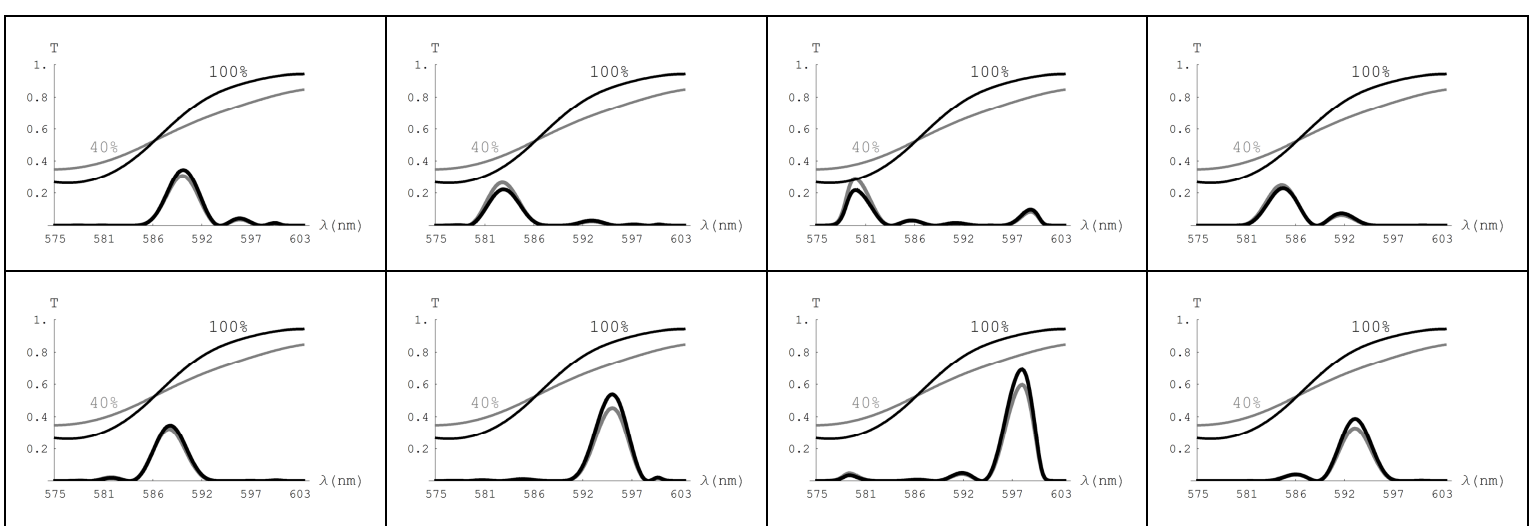

Figure 8. IRIS passbands as a function of oxygen saturation (black, 100\%; and grey, $40 \%$ ) for a $100 \mu \mathrm{m}$ vessel and modulated by Lambert-Beer's law. Note the isobestic point at $586 \mathrm{~nm}$ and its associated passbands.

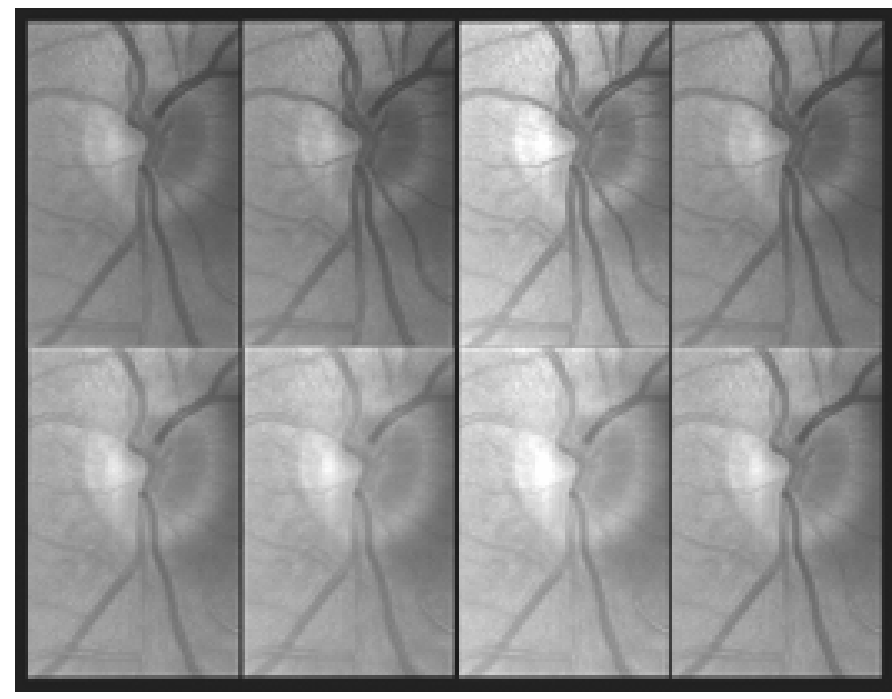

Figure 9. Eight narrow-band images of an optic disc recorded at the detector.

\section{CONCLUSIONS}

We have described two new techniques for spectral retinal imaging. The first one, a time sequintional technique enables a highly flexible approach to acquiring a wide-field spectral data cube for research and clinical exploration. We have shown that the use of vessel tracking and fitting of an analytical mathematical model to the transverse profiles suppresses the misregistration and calibration artefacts that are normally an issue for time-sequential techniques. Consequently, we have been able to perform qualitative and quantitative oxygen saturation measurements in the retinal vessel. The results obtained are in good agreement with those found in the literature. Future work using this instrument will assess clinical interpretation and refinement of the mathematical model for light propagation.

For clinical application a wide-field snapshot technique is desirable: the investigative data recorded here has informed the optimisation and design of the novel snapshot system spectral retinal imager. Multiple acquisition of spectral images in a single frame has been reported in the IRIS snapshot technique. The shape and number of IRIS spectral bands can be optimised for specific applications such as snapshot oximetry. The resulting optimised snapshot spectral retinal imager will enable enhanced biochemical measurements in the retina by eradicating calibration and misregistration problems associated with time-sequential techniques and the snapshot function is particularly valuable in a clinical setting. 


\section{REFERENCES}

1. A. R. Harvey, J. Beale, A. H. Greenaway, T. J. Hanlon and J. Williams, "Technology options for imaging spectrometry," Proc. SPIE 4132, 13-24 (2000).

2. M. R. Descour, C. E. Volin, E. L. Dereniak, K. J. Thome, A. B. Schumaker, D. W. Wilson, P. D. Maker, "Demonstration of a high speed non-scanning imaging spectrometer," Opt. Letters 22 (16), 1271-1273 (1997).

3. W. R. Johnson, D. W. Wilson, W. Fink, M. Humayun and G. Bearman, "Snapshot hyperspectral imaging in ophthalmology," J. Biomed. Opt. 12 (2007).

4. J. M. Beach, K. J. Schwenzer, S. Srinivas, D. Kim and J. S. Tiedeman, "Oximetry of retinal vessels by dualwavelength imaging: calibration and influence of pigmentation," J. Appl. Physiol 86, 748-758 (1999).

5. S. H. Hardarson, A. Harris, R. A. Karlsson, G. H. Halldorsson, L. Kagemann, E. Rechtman, G. M. Zoega, T. Eysteinsson, J. A. Benediktsson, A. Thorsteinsson, P. K. Jensen, J. Beach and Einar Stefánsson, "Automatic Retinal Oximetry," Investigative Ophthalmology and Visual Science 47, 5011-5016 (2006).

6. E. H. Aloni, A. Pollack, A. Grinvald, I. Vanzetta, D. A. Nelson, "Non-invasive imaging of retinal flow and oximetry by a new Retinal Function Imager," ARVO Abstract (2002).

7. A. R. Harvey, D. W Fletcher-Holmes, A. Gorman, K. Altenbach, J. Arlt and N. D Read, "Spectral imaging in a snapshot," Proc. SPIE 5694 (2005).

8. M. Hammer, D. Schweitzer, L. Leistritz, M. Scibor, K. Donnerhacke and J Strobel, "Imaging spectroscopy of the human ocular fundus in vivo," J. Biomed. Opt. 2 (4), 418-425 (1997).

9. N. Gat, "Imaging Spectroscopy Using Tuneable Filters: A Review," Proc. SPIE 4056, 50-64 (2000).

10. A. V. Cideciyan, "Registration of Ocular Fundus Images," IEEE Engineering in medicine and biology (1995).

11. J. B. Hickam, R. Frayser, J. C. Ross, "A study of retinal venous blood oxygen saturation in human subjects by photographic means," Circulation 27, 375-385 (1963).

12. F. C. Delori, "Non-invasive techniques for oximetry of blood in retinal vessels," Appl. Opt. 27, 1113-1125 (1988).

13. D. Schweitzer, L. Leistritz, M. Hammer, U. Batsch, M. Scibor, J. Kraft, C. Munch, W. Vilser, E. Bareshova.and P. Putsche, "Simultaneous measurement of oxygen saturation and vessel diameter by imaging spectrometry," Proc. SPIE 2930 (1996).

14. C. Chang, Hyperspectral Imaging: Techniques for Spectral Detection and Classification, Kluwer Academic/Plenum Publishers, New York, 2003.

15. Van Assendelft, Spectrophotometry of haemoglobin derivatives, Charles C. Thomas, Springfield, IL, 1970.

16. M. H. Smith, K. R. Denninghoff, A. Lompado and L. W. Hillman, "Effect of multiple light paths on retinal vessel oximetry," Appl. Opt. 39 (7), 1183-1193 (2000).

17. J. A. Sethian, Level Set Methods and Fast Marching Methods. Cambridge University Press, Cambridge, Massachusetts, 1999.

18. A. R. Harvey I. Alabboud,Al. Gorman, A. McNaught, S. Ramachandran and E. Theofanidou, "Spectral imaging of the retina," Proc. SPIE 6047 (2006).

19. A. R. Harvey, D. W Fletcher-Holmes, A. Gorman, K. Altenbach, J. Arlt and N. D Read, "Spectral imaging in a snapshot," Proc. SPIE 5694 (2005).

20. Discam: Stereoscopic optic disc camera from Marcher Enterprises Ltd. (UK).

21. I. Alabboud, D. Mordant, A. McNaught, A. R. Harvey , "Quantitative spectral imaging of the retina", ARVO Abstract (2007). 